\title{
Simulation numérique du déferlement en profondeur finie par méthode VOF
}

\author{
Stephan Guignard ${ }^{1,2}$, Richard Marcer ${ }^{2}$, Vincent Rey ${ }^{3}$, Christian \\ Kharif $^{3}$ \\ 'LSEET, Upresa 6017 du CNRS, Université Toulon-Var, BP 132, \\ 83957 La Garde Cedex, France \\ ${ }^{2}$ Principia RD S.A, Z. I. Brégaillon, 83507 La Seyne-sur-mer Cedex, France \\ ${ }^{3}$ IRPHE, Case 903, 163, avenue de Luminy, 13288 Marseille Cedex 9, France
}

\section{Résumé:}

En mer Méditerranée, le processus dominant à l'origine de l'érosion côtière est le déferlement de la houle. Nous avons simulé numériquement le déferlement d'une vague solitaire à partir du code de mécanique des fluides EOLE de la société PRINCIPIA. Ce modèle tridimensionnel en maillage curviligne multidomaines utilise une méthode VOF (Volume Of Fluids), particulièrement performante pour les problèmes à surface libre fortement déformée.

Nous présentons ici les résultats montrant la déformée de la surface libre et les champs de vitesses et de pression dans la zone de shoaling puis de déferlement, pour des ondes solitaires normalement incidentes sur des plages d'inclinaisons données. Ces résultats sont ensuite comparés avec ceux d'un modèle numérique basé sur des méthodes intégrales aux frontières (BIEM), particulièrement précis hors zone de déferlement.

\section{Introduction}

La modélisation du déferlement de la houle au dessus d'un fond variable ou sur une structure doit permettre d'en déduire les efforts subis par des ouvrages, à partir des champs de pression, et de préciser les conditions de mise en suspension de sédiments, directement liées aux champs de vitesses au voisinage du fond. La connaissance de l'évolution de la hauteur significative de la houle à l'approche de la plage avant, puis après déferlement, est essentielle (avec l'angle d'incidence) pour une bonne prise en compte des forçages de la houle dans les modèles de circulation côtière (équations de Saint-Venant).

Les obstacles majeurs rencontrés dans la modélisation numérique de la houle au voisinage du déferlement sont d'une part, l'importance croissante des effets non-linéaires jusqu'au déferlement, et d'autre part, le caractère diphasique du déferlement, où deux fluides de densités très différentes (l'air et l'eau) doivent être considérés. A ce stade, la définition d'une interface devient délicate.

Des modèles numériques à surface libre, précis et efficaces, et basés sur des méthodes intégrales aux frontières (BIEM), décrivent précisément la transformation de la houle à l'approche de la côte [Grilli, 1997]. Ces modèles, s'ils appréhendent très précisément les houles très cambrées rencontrées en eau très 
peu profonde, buttent sur le caractère rotationnel et visqueux de l'écoulement lors du déferlement ainsi que sur le fractionnement de la surface libre (emprisonnement de bulles, éjection de gouttes).

La méthode présentée ici prend en compte les effets de la viscosité en résolvant les équations de Navier-Stokes incompressibles par la méthode de pseudo-compressibilité. Le suivi d'interface est assuré par une méthode de type VOF autorisant les interfaces très cambrées et multiples, ainsi que le calcul des flux de quantité de mouvement à travers l'interface et donc la prise en compte de l'interaction dynamique entre les deux fluides. Elle est appliquée au cas d'ondes solitaires d'incidence normale sur des fonds de pentes constantes, qui sont une représentation correcte pour des ondes longues se propageant sur des fonds de pente faible (Peregrine, 1966; Yasuda et al, 1994).

\section{Le modèle}

Le modèle numérique est basé sur le code de mécanique des fluides EOLE de la société PRINCIPIA. Ce modèle tridimensionnel en maillage curviligne multidomaines utilise une méthode VOF (Volume Of Fluids), particulièrement performante pour les problèmes à surface libre fortement déformée, et est développé et appliqué ici au déferlement de vagues par profondeur finie.

\subsection{Equations de conservation et méthode de résolution}

Le probìme consiste à résoudre les équations de conservation de masse et de quantité de mouvement d'un fluide incompressible de masse volumique $\rho$, pour un écoulement bidimensionnel (2DV). Dans un repère cartésien xOy, d'axe vertical oy orienté vers le haut, les équations se résument matriciellement à:

$$
\frac{\partial W}{\partial t}+\frac{\partial F}{\partial x}+\frac{\partial G}{\partial y}=R
$$

avec

$$
W=\left[\begin{array}{l}
0 \\
\rho u \\
\rho v
\end{array}\right] ; F=\left[\begin{array}{l}
\rho u \\
\rho u^{2}+p+\tau_{x x} \\
\rho u v-\tau_{x y}
\end{array}\right] ; G=\left[\begin{array}{l}
\rho v \\
\rho u v+p+\tau_{y x} \\
\rho v^{2}-\tau_{y y}
\end{array}\right] ; R=\left[\begin{array}{l}
0 \\
0 \\
-\rho g
\end{array}\right]
$$

où $(u, v)$ sont les composantes du champ de vitesses, $p$ le champ de pression, $\tau_{\mathrm{i}, \mathrm{j}}$, $\mathrm{i}=1,2$ les composantes du tenseur des contraintes visqueuses et $\mathrm{g}$ l'accélération de la gravité. La résolution des équations précédentes utilise une méthode de pseudocompressibilité, particulièrement adaptée à la résolution des équations de NavierStokes [Viviand, 1995]. Le sytème précédent est transformé puis discretisé en volumes finis pour donner:

$$
\frac{\partial \widetilde{W}^{n+1}}{\partial \tau}+\frac{3 W^{n+1}-4 W^{n}+W^{n-1}}{2 \Delta t}+\left(\frac{\partial F}{\partial x}\right)^{n+1}+\left(\frac{\partial G}{\partial y}\right)^{n+1}=R^{n+1}
$$




$$
\begin{gathered}
\widetilde{W}^{n+1}=\left(\frac{\tilde{\rho}}{\rho}\right) W^{n+1} \\
P^{n+1}=\rho U_{0}^{2} \ln \left(\frac{\tilde{\rho}}{\rho}\right)+P_{0}
\end{gathered}
$$

où $\tilde{\rho}$ est la variable de pseudo-compressibilité et $\tau$ est le pseudo-temps.

C'est par un nombre d'itérations en pseudo-temps $\tau$ convenablement choisi par l'utilisateur et effectué entre deux itérations en temps $t$ que la solution de ce système converge vers la solution du système instationnaire à résoudre.

\subsection{Les conditions aux limites et le suivi d'interface}

Les parois sont supposées imperméables, les positions et mouvements des interfaces sont décrits par une méthode de type "Volume Of Fluid". Cette méthode consiste tout d'abord en la modélisation de l'interface par la fonction discrète $\mathrm{C}$ définie en chaque maille comme le taux d'occupation du fluide le plus dense dans la maille. L'évolution de $\mathrm{C}$ au cours du temps se déduit de l'équation de conservation de la masse traduite en terme de valeurs de $\mathrm{C}$ :

$$
\frac{\partial C}{\partial t}+\operatorname{div}(\vec{u} C)=0
$$

Le premier terme de cette équation est discrétisé temporellement grâce à un schéma d'ordre 1 , le deuxième terme, terme de flux, est calculé par la méthode de Hirt \& Nichols [1981].

\section{Les résultats:}

Nous avons étudié la propagation en eau peu profonde d'une vague de type "onde solitaire" qui permet d'adopter des conditions aux limites amont et aval très simples (vitesses nulles), tout en "modélisant" de façon très correcte l'aspect d'une houle très cambrée avant déferlement sur une pente faible [Yasuda, 1994]. La formulation choisie pour la détermination des champs de vitesses et de pression ainsi que de la forme de l'interface à l'instant initial est la solution exacte de l'onde solitaire [Tanaka, 1986]. Nous présentons ici le cas d'une onde d'amplitude initiale $0.5 \mathrm{~m}$ progressant tout d'abord sur un fond plat par une profondeur de $1 \mathrm{~m}$ puis sur un fond rectiligne incliné de pente $1 / 15$ (voir fig. 1).

\subsection{Evolution de la forme de l'interface:}

La forme de l'interface et l'amplitude de l'onde évoluent au cours de sa propagation vers la plage. C'est le phénomène de shoaling. L'évolution de l'amplitude est une donnée particulièrement importante (avec l'angle d'incidence, obtenu par ailleurs à partir de modèles de réfraction) pour exprimer en tout point les tensions de radiations, qui sont les termes moteurs dus à la houle qui apparaissent dans les équations de Saint-Venant (modèles de circulation 2DH). La figure 2 montre l'évolution de la forme de l'interface au cours du shoaling 
(respectivement aux temps $t=0.79 \mathrm{~s}, 3.40 \mathrm{~s}, 4.61 \mathrm{~s}$ et $5.08 \mathrm{~s}$ ), la figure 3 son évolution pendant le déferlement (respectivement aux temps $t=5.35 \mathrm{~s}, 5.43 \mathrm{~s}, 5.7 \mathrm{~s}$ et $5.71 \mathrm{~s}$ ), le point de déferlement étant défini comme l'instant où l'interface présente une tangente verticale en au moins un point. L'abscisse de déferlement est définie par la position de la crête à cet instant.

Les résultats concernant la variation de l'amplitude de la houle au cours de sa propagation, de sa hauteur au point de déferlement, et de la position du déferlement sont confrontés aux solutions obtenues pour différentes amplitudes initiales à partir de méthodes BIEM [Grilli, 1997].

La figure 4 montre pour les deux méthodes des évolutions similaires de la hauteur au cours de la propagation. Les figures 5 et 6 montrent en trait plein respectivement la hauteur de l'onde et la position de la crête au point de déferlement pour différentes hauteurs initiales de l'onde. Les résultats correspondant à la présente méthode pour une amplitude initiale de $0.5 \mathrm{~m}$ sont reportés sur ces mêmes figures, et montrent le bon accord $(7 \%$ d'erreur sur la hauteur au point de déferlement) avec les résultats utilisant les méthodes BIEM.

Dans tous les cas les résulitats obtenus par la méthode présentée ici sont tout à fait en accord avec les résultats obtenus par les méthodes BIEM qui font référence en la matière. Il est tout de même à noter un certain retard au déferlement (fig. 6) qui est dû au manque de précision temporaire du suivi d'interface utilisé.

\subsection{Vitesses et pressions au fond}

La connaissance des champs de pression et de vitesses au voisinage de l'interface fluide-solide est primordiale pour les calculs des efforts de houles sur des structures, et importante pour une meilleure paramétrisation de la quantité de matière mise en suspension par la houle lors de son déferlement et du transport qui en résulte par les courants générés dans cette zone. Les figure 7 et 8 présentent respectivement les intensités de vitesse horizontale et de pression totale au voisinage du fond, en deux positions données, la première, en $x=29.0 \mathrm{~m}$, étant située avant déferlement, la seconde, en $x=31.7 \mathrm{~m}$, juste après déferlement. Les résultats font apparaitre l'évolution rapide de ces champs dans cette zone, avec de fortes variations locales des intensités des vitesses et des pressions ce qui laisse présager des effets importants, d'un point de vue de l'impact sur les structures solides, et pour la mise en suspension rapide et importante de sédiments pour des côtes sablonneuses.

\section{Conclusions}

Nous avons présenté les résultats de notre modèle, en insistant plus particulièrement sur la déformée de la surface libre et les champs de vitesses et de pression dans la zone de shoaling puis de déferlement, pour des ondes solitaires normalement incidentes sur des plages d'inclinaisons données.

La comparaison avec les résultats obtenus à partir d'une méthode BIEM

- montre la très bonne aptitude de notre méthode à décrire le phénomène de 
shoaling jusqu'au déferlement. Les faibles écarts observés sont dus d'une part aux hypothèses inhérentes à chacun des deux modèles, et d'autre part pour notre modèle à la précision des calculs. La prise en compte des effets visqueux dans notre méthode (alors que la méthode BIEM suppose un fluide parfait) permet une meilleure représentation de la physique au voisinage du déferlement où ces effets croissent rapidement (forts gradients de vitesses au voisinage du fond), et reste surtout la seule applicable dans la zone où les mélanges eau-air sont importants.

La méthode de suivi d'interface doit encore être améliorée (développement actuel d'une méthode de calcul d'interface beaucoup plus précise), et le maillage raffiné pour pouvoir représenter correctement le comportement de la houle dans la zone de déferlement jusqu'à la plage. Les applications à des géométries plus complexes du fond sont envisagées, des géométries simples ont été choisies jusqu'ici pour la validation de notre méthode à partir de comparaisons avec des cas traités dans la littérature. Des expériences de mesure des vitesses de «surface » au voisinage du déferlement sont par ailleurs prévues pour valider notre méthode au niveau du déferlement où des modifications très rapides des vitesses apparaissent.

\section{Remerciements:}

Ce travail a été effectué dans le cadre du programme Européen Mast III FANS (Contract MAS3-CT95-0037), et du Programme National de l'Océanographie côtière (PNOC). Il bénéficie du soutien financier du CNRS et de Principia R\&D pour le financement de la thèse de Stéphan Guignard, boursier BDI-CNRS Entreprise.

\section{Références:}

S. T. Grilili, I. A. Svendsen and R. Subramanya, "Breaking criterion and characteristics for solitary waves on slopes ", I. of Waterway, Port, Coastal and Ocean Eng. May-June 1997, pp 102-112.

C. W. Hirt and B. D. Nicholls, "Volume Of Fluid methods for the dynamics of free boundaries", J. Comp. Phys. 39, pp 323-345 (1981).

T. Yasuda, S. Shinoda and T. Hattori, "Soliton-mode wavemaker theory and system for coastal waves", Proc. XXIVth ICCE, pp 704-718 (1994).

D. H. Peregrine "Long waves on beaches", J. Fluid Mechanics. 27 (4), pp 815-827 (1966).

H. Viviand, "Analysis of pseudo-compressibility systems for compressible and uncompressible flows", Comp. Fluid. Dynamics Rev. 29 (3), pp 650-655 (1995).

T. Yasuda, S. Shinoda and T. Hattori, "Soliton-mode wavemaker theory and system for coastal waves", Proc. XXIVth ICCE, pp 704-718 (1994). 


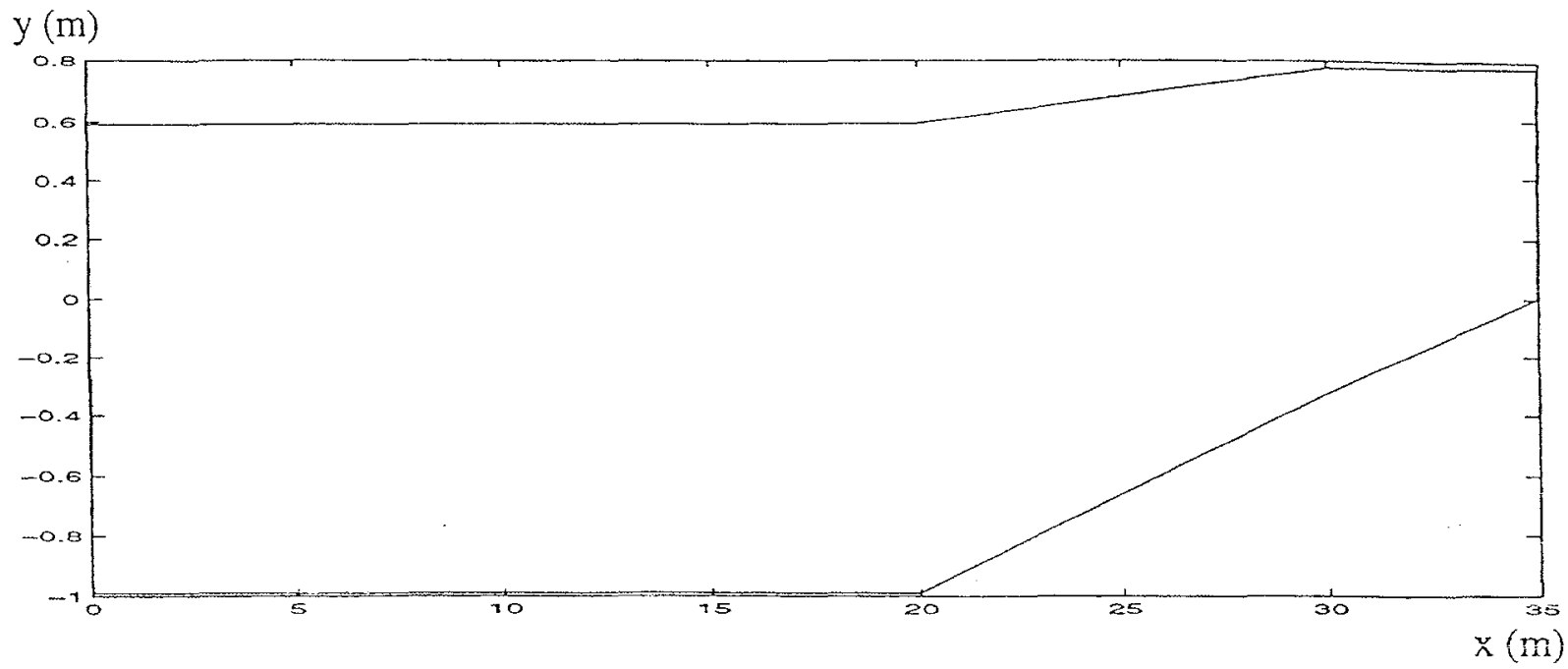

fig 1: La boite de calcul

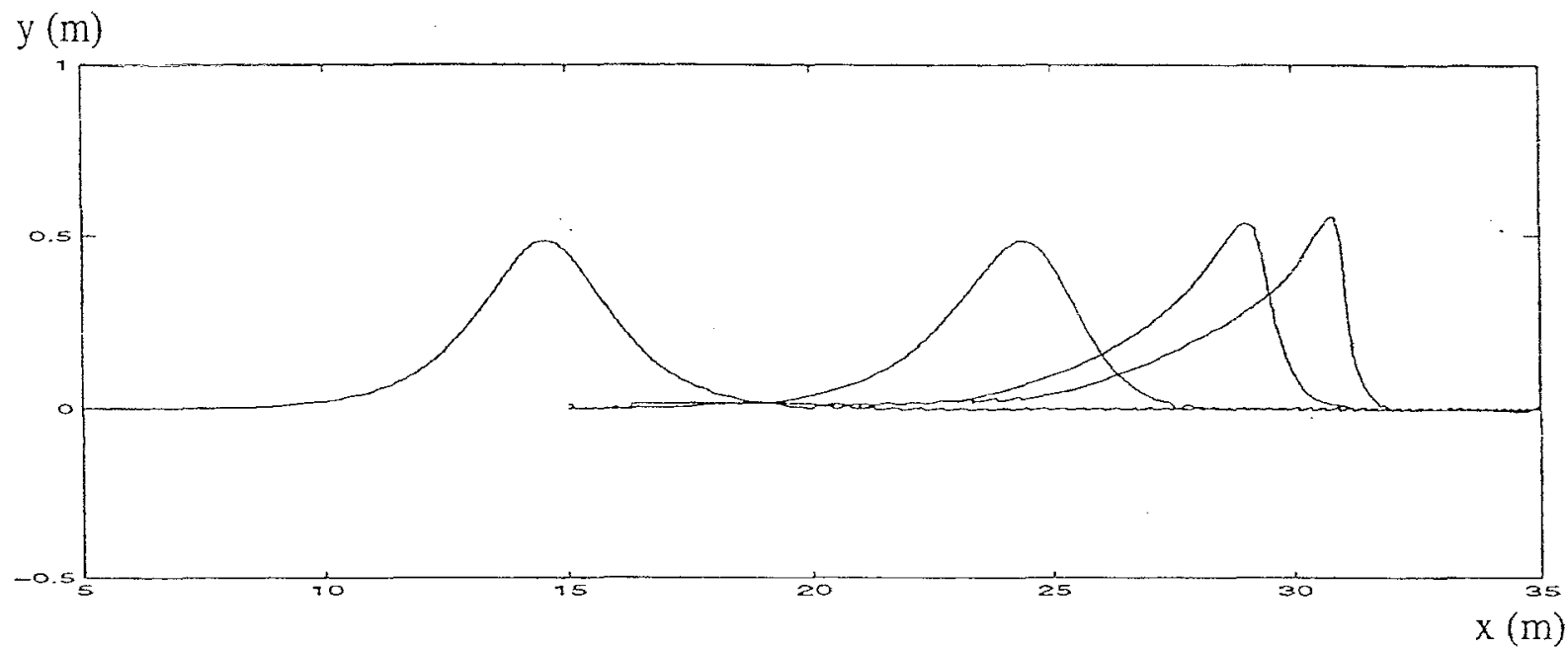

fig2: Evolution de l'interface avant le déferlement

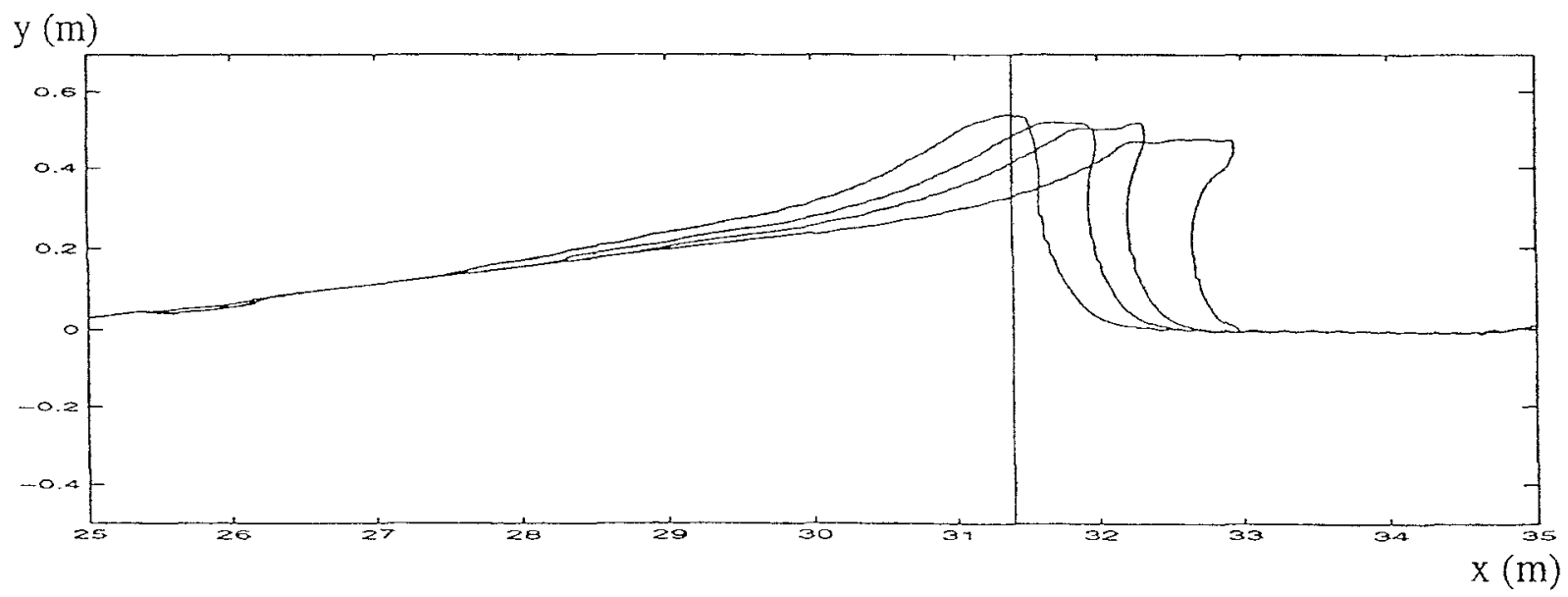

fig 3 : Evolution de l'interface pendant le déferlement 


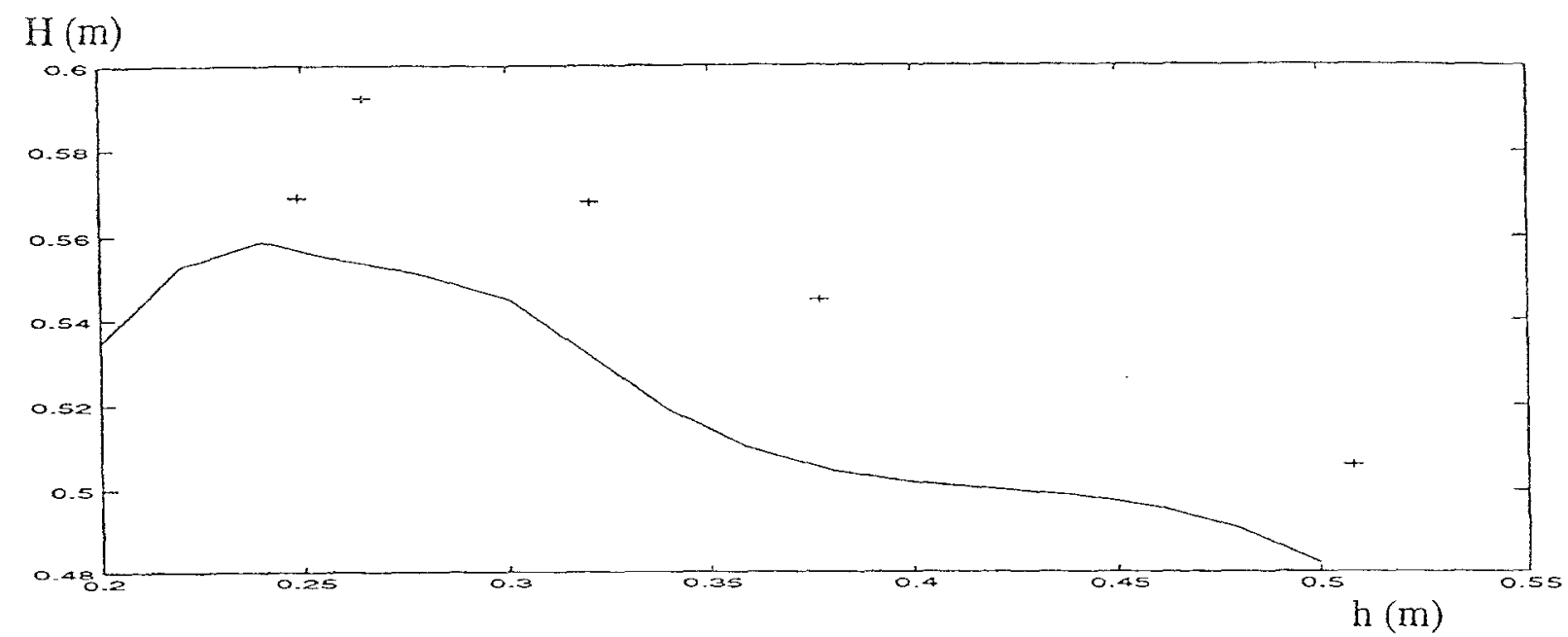

fig4: Hauteur de crête en fonction de la profondeur sous la crête : — : Méthode BIEM $+\quad$ : Méthode VOF

$\mathrm{Hd}(\mathrm{m})$

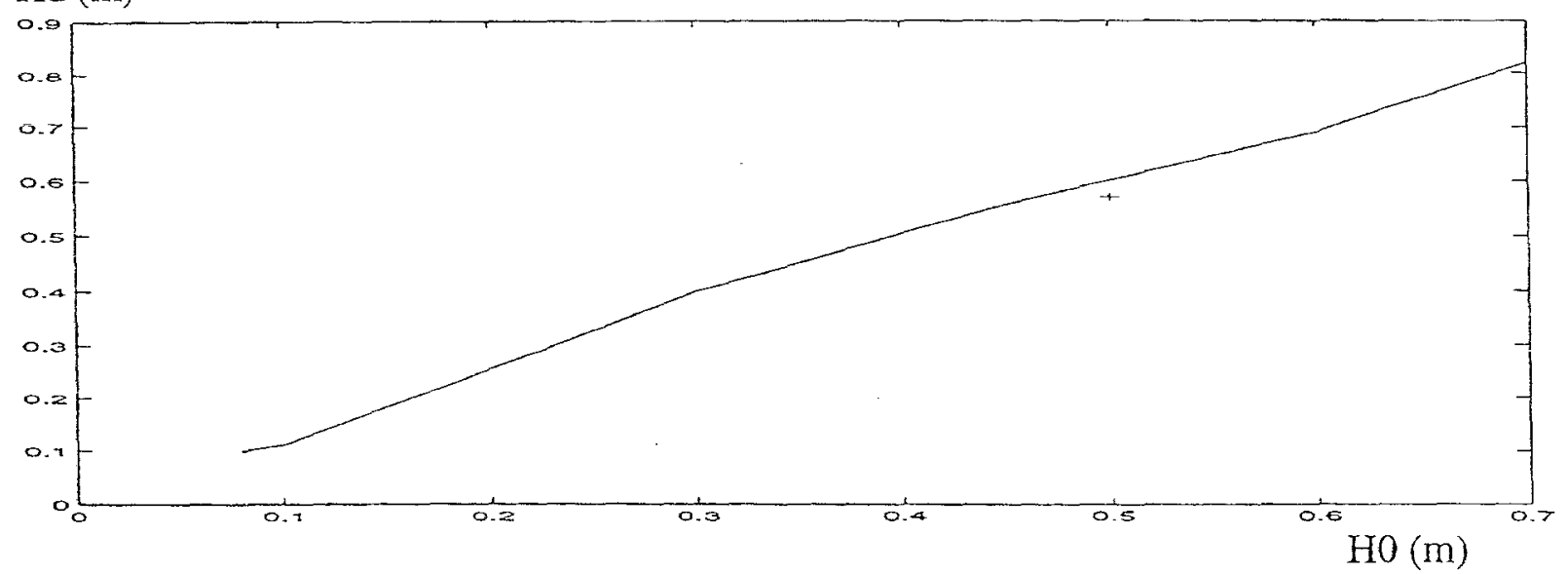

fig5: Hauteur au déferlement en fonction de la hauteur initiale : — - : Méthode BIEM

$+\quad$ : Méthode VOF

$\mathrm{xd}(\mathrm{m})$

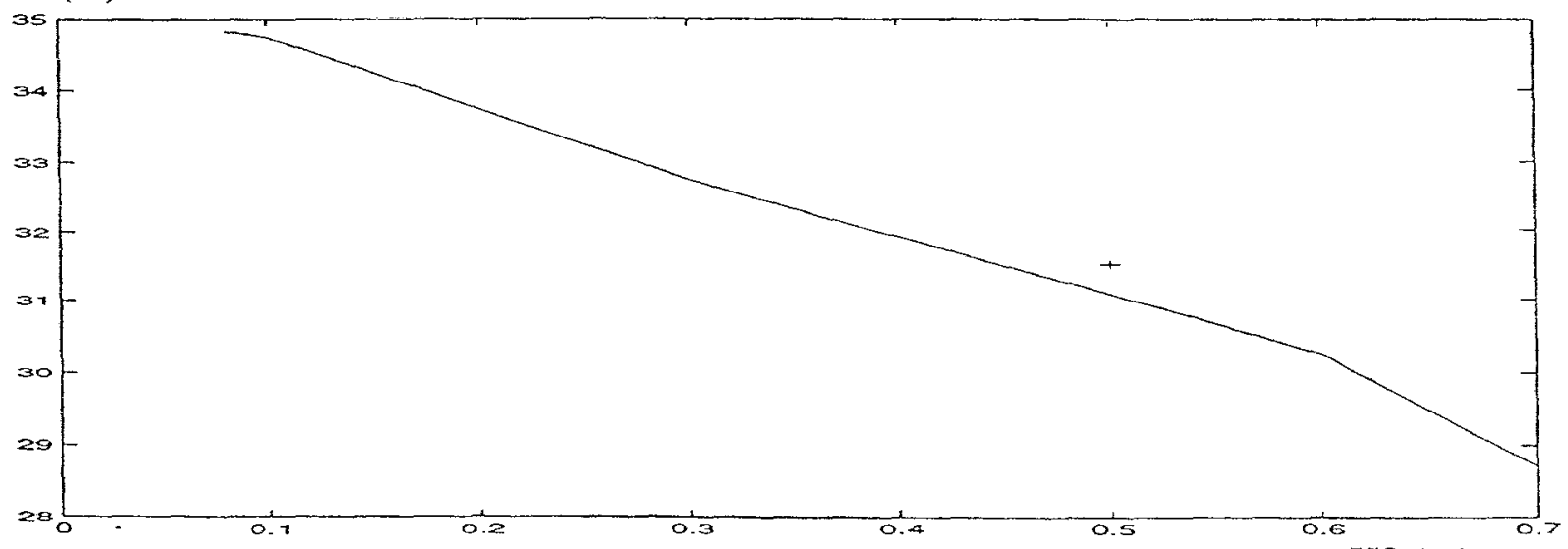

$\mathrm{HO}(\mathrm{m})$

fig6: Position de la crête au déferlement en fonction de la hauteur initiale : 
Ufond $(\mathrm{m} / \mathrm{s})$

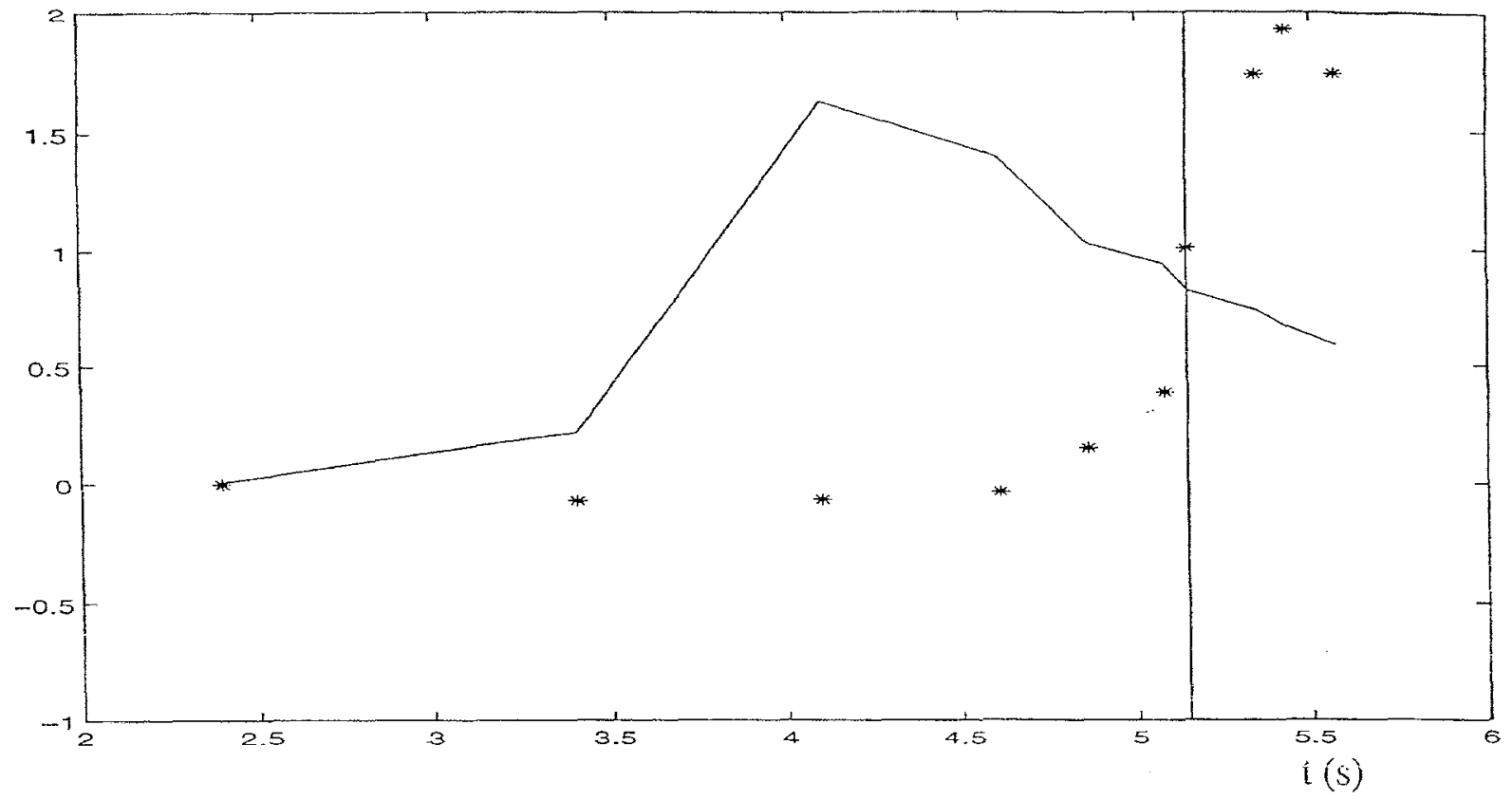

fig7 : vitesse au fond pour : $x=29 \mathrm{~m}$ (Hors déferlement) : $\mathrm{x}=31,7 \mathrm{~m}$ (zone de déferlement) : *

Pfond-pression initiale ( $\mathrm{Pa})$

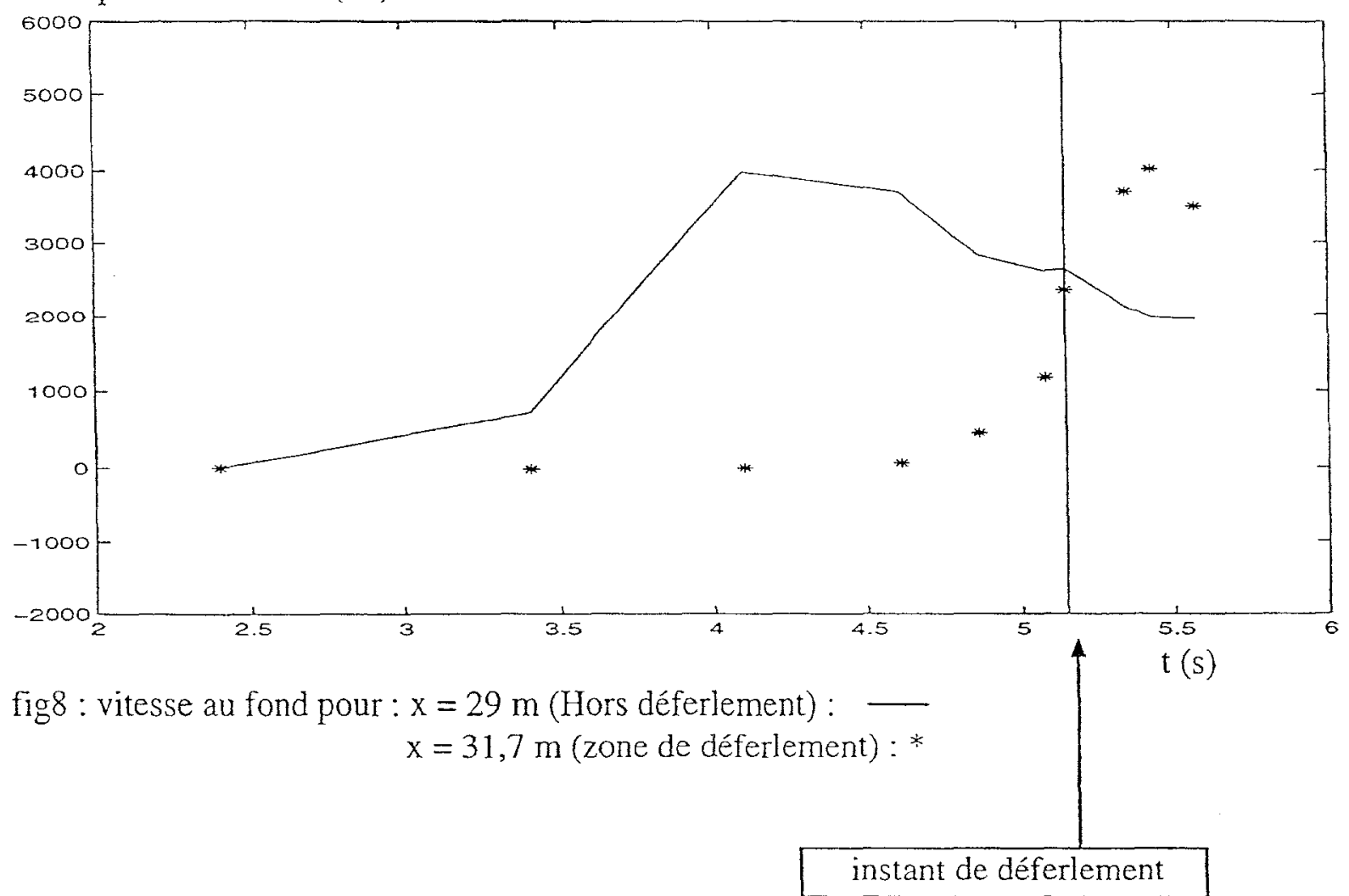

